\title{
Preferences in the management of high-risk prostate cancer among urologists in Europe: results of a web-based survey
}

\section{Cristian I. Surcel* , Prasanna Sooriakumaran ${ }^{\dagger \ddagger}$, Alberto Briganti§, Pieter J.L. De Visschere $\$ \S$, Jurgen J. Fütterer ${ }^{\dagger \dagger \dagger}$, Pirus Ghadjar ${ }^{\ddagger \ddagger}$, Hendrik Isbarn ${ }^{\text {} T}$, Piet Ost** , Guillaume Ploussard ${ }^{\ddagger \ddagger \ddagger}$,

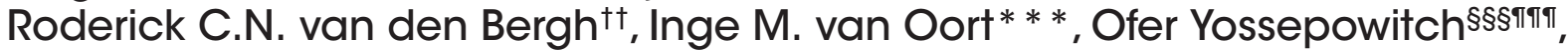 J.P. Michiel Sedelaar*** and Gianluca Giannarini"; Members of the Prostate Cancer Working Group of the Young Academic Urologists Working Party and Members of the Young Urologists Office of the European Association of Urology}

\begin{abstract}
*Centre of Uronephrology and Renal Transplantation, Fundeni Clinical Institute, "Carol Davila" University of Medicine and Pharmacy, Bucharest, Romania, 'Surgical Intervention Trials Unit, Nuffield Department of Surgical Sciences, University of Oxford, Oxford, UK, ${ }^{\ddagger}$ Department of Molecular Medicine and Surgery, Karolinska Institute, Stockholm, Sweden, §Department of Urology, Urological Research Institute, Vita-Salute University San Raffaele, Milan, "Department of Experimental and Clinical Medical Sciences, Urology Unit, University of Udine, Udine, Italy, \$sDepartment of Radiology, * Department of Radiation Oncology and Experimental Cancer Research, Ghent University Hospital, Ghent, Belgium, ${ }^{\dagger+}$ Department of Radiology, ${ }^{* * *}$ Department of Urology, Radboud University Medical Centre, Nijmegen, ${ }^{+\dagger}$ Department of Urology, University Medical Centre Utrecht, Utrecht, The Netherlands, ${ }^{\ddagger \ddagger}$ Department of Radiation Oncology, Charité Universitätsmedizin Berlin, Berlin, " "Department of Urology, University Medical Center Hamburg-Eppendorf, Hamburg, Germany, ${ }^{\ddagger \neq \ddagger}$ Department of Urology, CHU Saint-Louis, Assistance Publique - Hôpitaux de Paris, Université Paris Est Creteil, Paris, France and $\S^{\S}$ Department of Urology, Rabin Medical Center - Beilinson, Petach-Tikva, "TाT'Sackler Faculty of Medicine, University of Tel Aviv, Tel Aviv, Israel
\end{abstract}

\section{Objective}

To explore preferences in the management of patients with newly diagnosed high-risk prostate cancer (PCa) among urologists in Europe through a web-based survey.

\section{Materials and Methods}

A web-based survey was conducted between 15 August and 15 September 2013 by members of the Prostate Cancer Working Group of the Young Academic Urologists Working Party of the European Association of Urology (EAU). A specific, 29-item multiple-choice questionnaire covering the whole spectrum of diagnosis, staging and treatment of high-risk $\mathrm{PCa}$ was e-mailed to all urologists included in the mailing list of EAU members. Europe was divided into four geographical regions: Central-Eastern Europe (CEE), Northern Europe (NE), Southern Europe (SE) and Western Europe (WE). Descriptive statistics were used. Differences among sample segments were obtained from a z-test compared with the total sample.

\section{Results}

Of the 12850 invited EAU members, 585 urologists practising in Europe completed the survey. High-risk PCa was defined as serum PSA $\geq 20 \mathrm{ng} / \mathrm{mL}$ or clinical stage $\geq \mathrm{T} 3$ or biopsy Gleason score $\geq 8$ by $67 \%$ of responders, without significant geographical variations. The preferred single-imaging examinations for staging were bone scan $(74 \%, 81 \%$ in WE and $70 \%$ in SE; $P=0.02$ for both), magnetic resonance imaging (53\%, $72 \%$ in WE and $40 \%$ in SE; $P=0.02$ and $P=$ 0.01 , respectively) and computed tomography (45\%, $60 \%$ in SE and $23 \%$ in WE; $P=0.01$ for both). Pre-treatment predictive tools were routinely used by $62 \%$ of the urologists, without significant geographical variations. The preferred treatment was radical prostatectomy as the initial step of a multipletreatment approach $(60 \%, 40 \%$ in NE and $70 \%$ in CEE; $P=0.02$ and $P<0.01$, respectively), followed by external beam radiation therapy with androgen deprivation therapy (29\%, 45\% in NE and 20\% in CEE; $P=0.01$ and $P=0.02$, respectively), and radical prostatectomy as monotherapy (4\%, $7 \%$ in WE; $P=0.04$ ). When surgery was performed, the open retropubic approach was the most popular $(58 \%, 74 \%$ in CEE, $37 \%$ in NE; $P<0.01$ for both). Pelvic lymph node dissection was performed by $96 \%$ of urologists, equally split between a standard and extended template. There was no consensus on the definition of disease recurrence after primary treatment, and much heterogeneity in the administration of adjuvant and salvage treatments.

\section{Conclusion}

With the limitation of a low response rate, the present study is the first survey evaluating preferences in the management of high-risk PCa among urologists in Europe. Although the 
definition of high-risk PCa was fairly uniform, wide variations in patterns of primary and adjuvant/salvage treatments were observed. These differences might translate into variations in quality of care with a possible impact on ultimate oncological outcome.

\section{Keywords}

prostatic neoplasms, prostatectomy, radiation therapy, androgen deprivation therapy, high-risk prostate cancer, survey

\section{Introduction}

High-risk prostate cancer (PCa) accounts for up to $40 \%$ of newly diagnosed cases, depending on the definition used [1,2]. Despite refinement in patient selection and advances in primary and adjuvant therapies, disease recurrence remains substantial, affecting $>50 \%$ of patients within 10 years after treatment and carrying a significant risk of progression and death $[1,3]$.

In the absence of high-level evidence, the best management approach for patients with high-risk PCa is still under debate. Guidelines from several scientific societies $[4,5]$ provide useful information, but evidence as to compliance to these is scarce and conflicting [6,7]. All too often, therefore, the ultimate management of high-risk PCa is influenced by the individual preference of the treating urologist, the presence of a multidisciplinary institutional team, local availability of diagnostic and therapeutic technology and other non-medical factors, such as patient socio-economic status, geographical area and medicolegal issues [8-10].

There remains a paucity of data on attitudes of treating urologists in the contemporary PCa literature. In the present study, we explored preferences in the management of patients with newly diagnosed high-risk PCa among urologists practising in Europe through a web-based survey.

\section{Materials and Methods}

On 15 August 2013, a specific web-based questionnaire created on surveymonkey.com by members of the Prostate Cancer Working Group of the Young Academic Urologists Working Party of the European Association of Urology (EAU) was sent by e-mail to 12850 urologists who were included in the mailing list of the EAU members.

Participants were invited to answer a multiple-choice questionnaire including 29 items covering the whole spectrum of diagnosis, staging and treatment of high-risk PCa. Multiple responses were allowed only for a few questions (Appendix S1).

Data collection was stopped 30 days later, on 15 September 2013. All data were processed and analysed using $\mathrm{GfK}^{\odot}$ Romania (GfK Group, Nuremberg, Germany) in accordance with the International Chamber of Commerce/European Society for Opinion and Market Research Code on Market and Social Research [11]. To evaluate whether differences were related to the geographical origin of respondents, Europe was divided into four areas: Central Eastern Europe (CEE), Northern Europe (NE), Southern Europe (SE) and Western Europe (WE [Appendix S2]).

Descriptive statistics were used. Differences among sample segments were obtained from a z-test compared with total sample, performed at a 95\% confidence level. A $\pm 3.9 \%$ sampling error was observed. All reported $P$ values were two-sided and statistical significance was set at $P=0.05$. SPSS 21.0 software was used for statistical analysis.

\section{Results}

Of the 12850 invited EAU members, 9829 practised in European countries, and 8234 were urologists. Of these, 585 (7\%) completed the survey, 105 (18\%) from CEE, 36 (6\%) from NE, 281 (48\%) from SE and 163 (28\%) from WE.

Of the 585 urologists, 246 (42\%) were based at an academic hospital (56\% in CEE and $28 \%$ in WE; $P=0.01$ and $P<0.01$, respectively), and $153(26 \%)$ practised in a regional hospital ( $43 \%$ in WE and $11 \%$ in CEE; $P=0.01$ and 0.02 , respectively). Of the respondents, 293 (50\%) had $>10$ years of experience in PCa management, and 223 (38\%) were high-volume surgeons, defined as performing $>50$ radical prostatectomies (RPs) per year. The vast majority of respondents $(498 / 585,85 \%)$ worked within a multidisciplinary team, mostly including oncologists, radiation oncologists and radiologists, this percentage being the highest in WE (98\%) and the lowest in CEE (77\%; $P=0.01$ for both).

\section{Definition and Clinical Staging}

High-risk PCa was defined as PSA $\geq 20 \mathrm{ng} / \mathrm{mL}$ or clinical stage $\geq$ T3 or biopsy Gleason score $\geq 8$ by the majority of respondents $(392 / 585,67 \%)$, with no significant geographical variations (Fig. 1).

The most commonly performed imaging examinations for clinical staging were bone scan $(74 \%, 81 \%$ in WE and $70 \%$ in SE; $P=0.02$ for both), MRI (53\%, 72\% in WE and $40 \%$ in SE; $P=0.02$ and $P=0.01$, respectively) and CT $(45 \%, 60 \%$ in SE and $23 \%$ in WE; $P=0.01$ for both). Pre-treatment predictive tools were used on a routine basis by $62 \%$ of the respondents, without significant geographical variations. Partin tables and 
Fig. 1 Preferences for definition of high-risk prostate cancer. Numbers are proportions of respondents per geographical area. Total refers to the entire European sample $(N=585)$. PFS, progression-free survival.

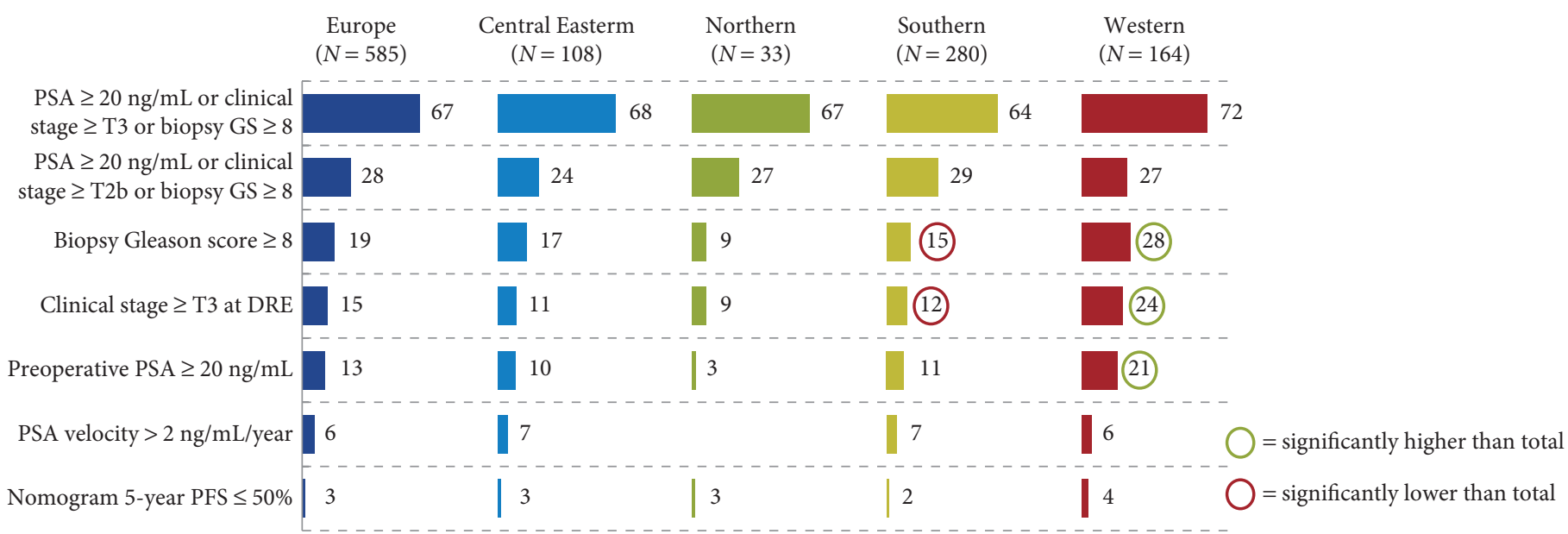

Fig. 2 Preferences for initial management of high-risk prostate cancer. Numbers are proportions of respondents per geographical area. Total refers to the entire European sample $(N=585)$. EBRT, external beam radiation therapy.

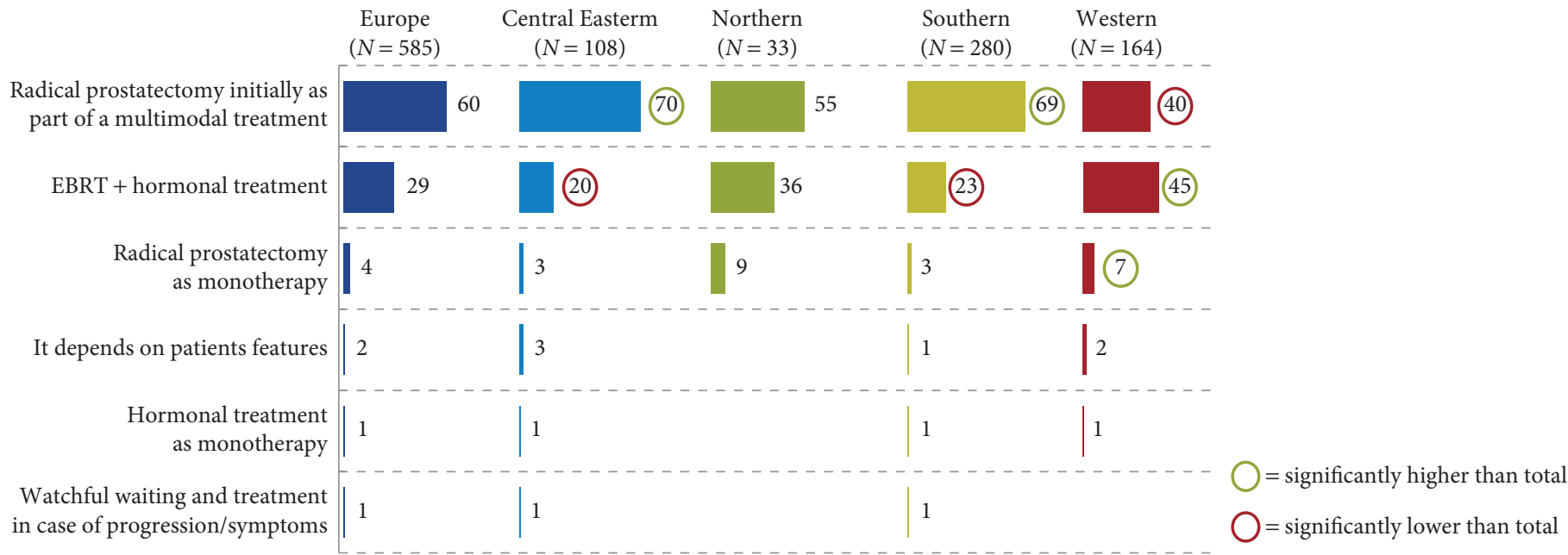

D'Amico risk classification were the most frequently used (50 and $41 \%$, respectively, without significant geographical variations).

\section{Initial Treatment}

The preferred initial treatment was RP as part of a multiple-treatment approach for $60 \%$ of the respondents $(70 \%$ in CEE and $40 \%$ in WE; $P=0.02$ and $P<0.01$, respectively), followed by external beam radiation therapy (EBRT) with androgen deprivation therapy (ADT) by $29 \%$ (45\% in NE and $20 \%$ in CEE; $P=0.01$ and $P=0.02$, respectively), and RP as monotherapy by $4 \%$ (7\% in WE; $P=0.04$ [Fig. 2]).

When treatment was RP, the open retropubic approach was the most frequently adopted (58\% overall, $74 \%$ in CEE and $37 \%$ in WE; $P<0.01$ for both [Fig. 3]). Attempted nerve-sparing was systematically avoided by $60 \%$ of the respondents (73\% in SE and $47 \%$ in CEE; $P=0.02$ for both).

Pelvic lymph node dissection (PLND) was routinely performed by $96 \%$ of respondents (98\% in SE and $88 \%$ in NE; $P=0.03$ and $P=0.02$, respectively). A standard (i.e. external, internal and obturator nodes bilaterally) and extended (i.e. common iliac, external, internal and obturator nodes bilaterally) dissection template was equally adopted (31 and $30 \%$ of respondents, respectively), with no significant geographical variations (Fig. 4). Intra-operative frozen sections during PLND were routinely performed by $13 \%$ of the respondents ( $19 \%$ in CEE; $P=0.03$ ), and $46 \%$ of respondents (63\% in WE; $P=0.02$ ) abandoned RP if lymph node invasion 


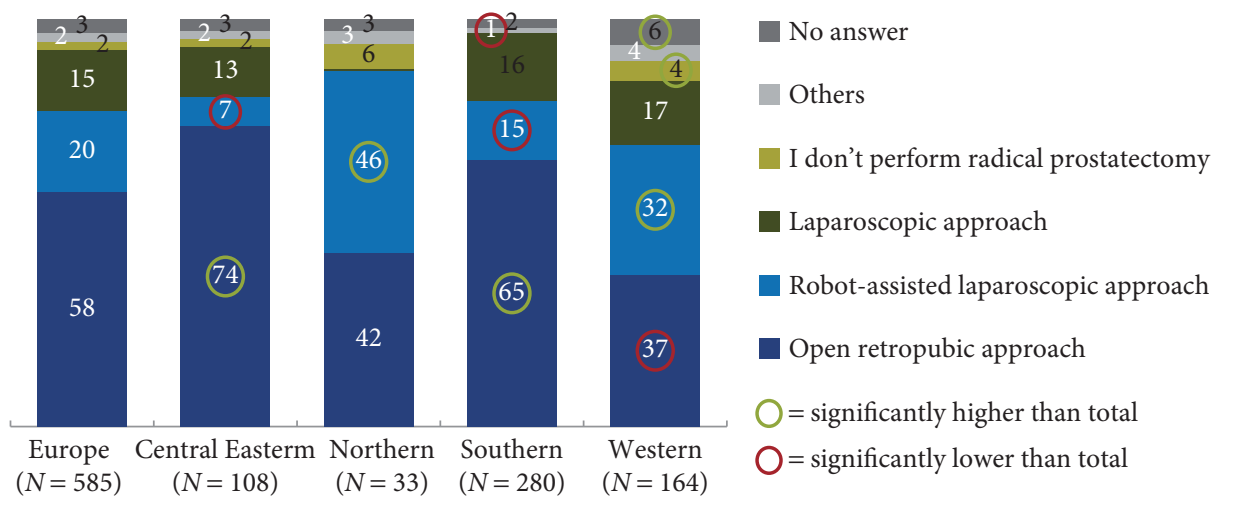

Fig. 3 Preferences for radical prostatectomy approach for high-risk prostate cancer. Numbers are proportions of respondents per geographical area. Total refers to the entire European sample $(N=585)$.

Fig. 4 Preferences for pelvic lymph node dissection template at the time of radical prostatectomy for high-risk prostate cancer. Numbers are proportions of respondents per geographical area. Total refers to the entire European sample $(N=585)$. LNI, lymph node invasion.

Europe Central Easterm Northern
bilaterally, in all cases

was proven. Regarding the minimal nodal yield required for an accurate PLND, opinions varied, with some respondents (56\%) considering that the minimum number should exceed 10, and others (37\%) judging that dissection of an anatomically defined pelvic region was more important than lymph node count.

\section{Definition and Treatment of Disease Recurrence}

Biochemical recurrence (BCR) after RP was defined as any rise in PSA level $\geq 0.2 \mathrm{ng} / \mathrm{mL}$, confirmed with a second measurement at least 1 month apart, by $47 \%$ of the respondents, while $26 \%$ defined BCR as three consecutive rises of PSA $\geq 0.2 \mathrm{ng} / \mathrm{mL}$ (Fig. 5). No significant geographical variations were observed.

Regarding the definition of local recurrence after RP, responses varied from a positive biopsy from anastomosis site/pelvis (32\%) to abnormal findings on MRI (29\%) and to positive surgical margins (24\%), with no significant geographical variations (Fig. 6).

In all, $47 \%$ of the respondents defined BCR after EBRT as any rise in PSA level $\geq 2 \mathrm{ng} / \mathrm{mL}$ above the post-treatment nadir, while $37 \%$ of respondents defined it as three consecutive PSA rises above the post-treatment nadir. No significant geographical variations were observed.

Concerning adjuvant/salvage treatment after RP, $53 \%$ of respondents (63\% in SE and 36\% in NE; $P=0.02$ and $P=0.01$, respectively) offered adjuvant EBRT only if surgical margins were positive, most often within 3 months after surgery, while $43 \%$ (51\% in WE; $P=0.02$ ) recommended salvage EBRT in the case of BCR (Fig. 7). Adjuvant ADT was offered only if lymph nodes were positive by $62 \%$ of respondents ( $73 \%$ in SE, $45 \%$ in WE; $P=0.01$ for both), while salvage ADT was offered 
Fig. 5 Preferences for definition of biochemical recurrence after radical prostatectomy for high-risk prostate cancer. Numbers are proportions of respondents per geographical area. Total refers to the entire European sample $(N=585)$.

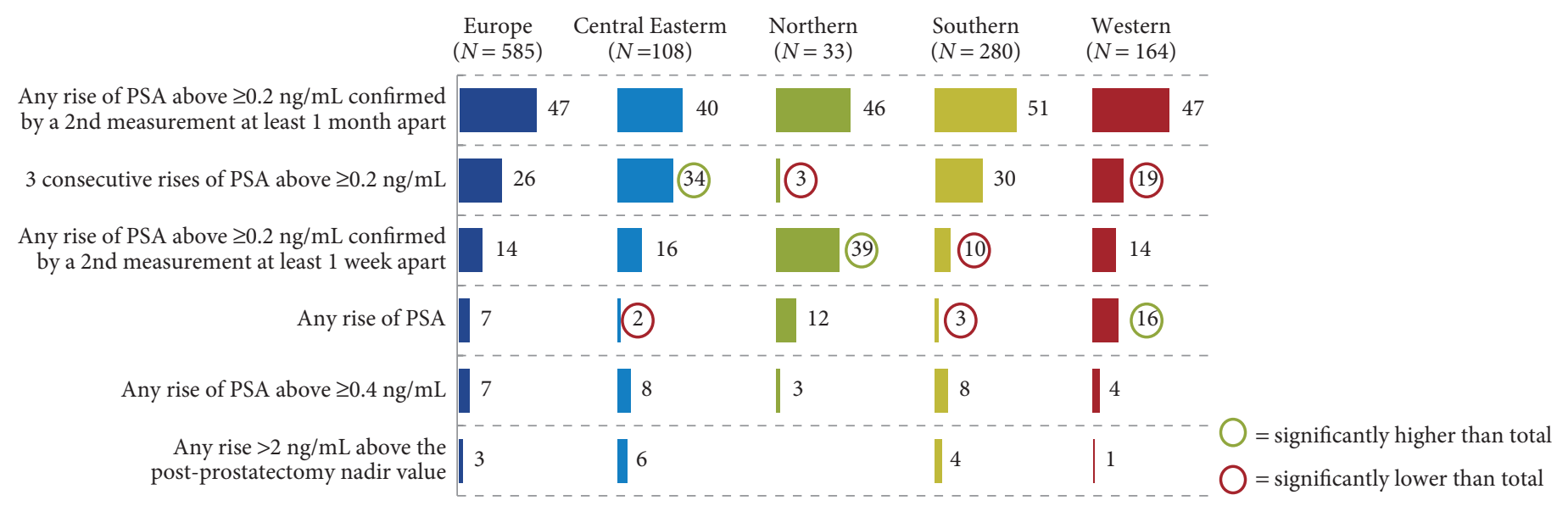

Fig. 6 Preferences for definition of local recurrence after radical prostatectomy for high-risk prostate cancer. Numbers are proportions of respondents per geographical area. Total refers to the entire European sample $(N=585)$. PET, positron emission tomography.

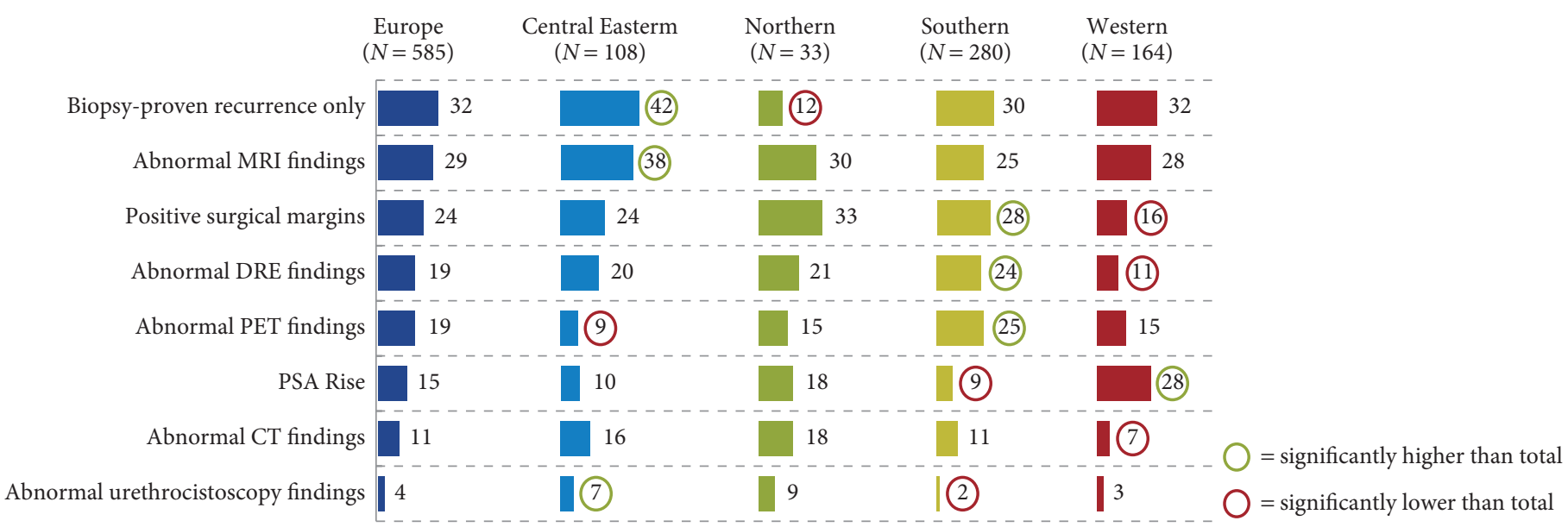

in the case of BCR and distant metastases by $39 \%$ (53\% in CEE, 26\% in WE; $P=0.02$ for both) and $76 \%$ (without significant geographical variations) of respondents, respectively (Fig. 8).

\section{Discussion}

The present study is the first international survey evaluating preferences in the management of high-risk PCa among urologists in Europe. There was good agreement on the definition of high-risk disease. The preferred treatment was found to be open RP with either standard or extended PLND as the initial step of a multiple-treatment approach. Consensus on the definition of disease recurrence after primary treatment was weaker, and there was much heterogeneity in administration of adjuvant and salvage therapies. Geographical differences were observed with regard to several questionnaire items.
Several results of our survey may be of interest. First, the definition of high-risk PCa was shared by $\sim 66 \%$ of the respondents. The most commonly used definition was any cancer with PSA $\geq 20 \mathrm{ng} / \mathrm{mL}$ or clinical stage $\geq \mathrm{T} 3$ or biopsy Gleason score $\geq 8$, which represents the definition published in both the EAU and National Comprehensive Cancer Network (NCCN) guidelines [4,5].This should facilitate the conduct of multinational collaborations for trials on high-risk PCa.

Second, the majority of respondents would offer RP as the initial step of a multiple-treatment approach for PCa. While EBRT with long-term ADT has long been considered the standard of care compared with other treatment methods, over the past decade there has been a surge in the use of RP as primary treatment in selected patients, followed by EBRT in case of adverse pathological features [12]. Both the EAU and NCCN guidelines [5] recommend radiation therapy with ADT 
Fig. 7 Preferences for administration of $\mathbf{A}$, adjuvant and B, salvage external beam radiation therapy after radical prostatectomy for high-risk prostate cancer. Numbers are proportions of respondents per geographical area. Total refers to the entire European sample $(N=585)$.

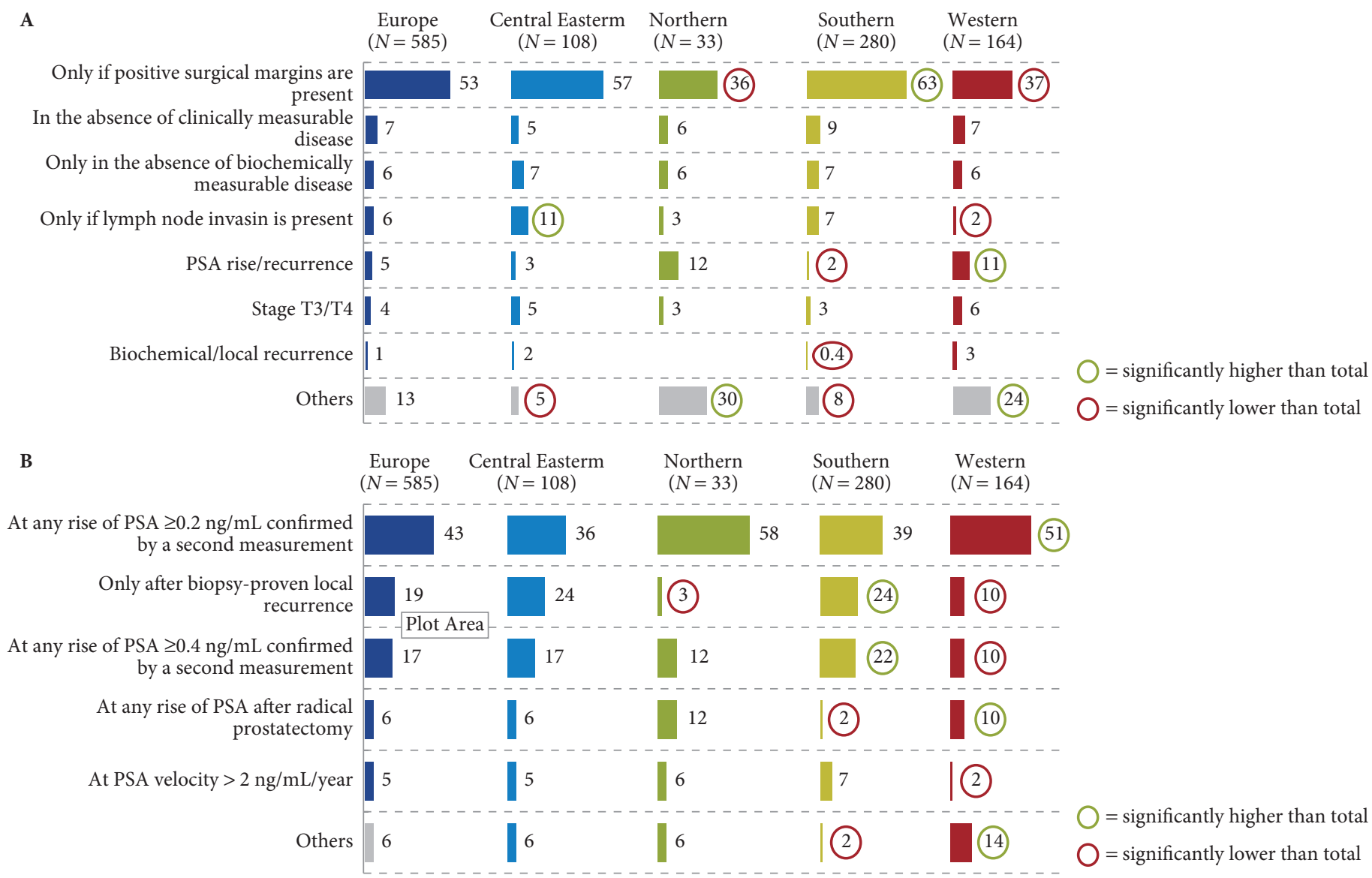

as the preferred treatment for high-risk PCa, and indicate surgery as an option to selected patients with low-volume disease [4] or no fixation to adjacent organs [5]. The findings in the present survey may reflect data from recent population-based studies comparing surgery and EBRT for clinically localized $\mathrm{PCa}$, which have consistently demonstrated a possible benefit of surgery, especially in younger and fitter patients with more aggressive disease [13-16]; however, the target population of our survey were urologists only, which may have biased the choice of treatment towards surgery.

Third, the preferred approach for RP is open (58\%), followed by robot-assisted $(20 \%)$ and laparoscopic (15\%). One reason might be the reluctance of urologists to use newer, i.e. robotic, technology in patients with an aggressive form of disease, a finding common to other settings [17]. In particular, the lack of tactile feedback with the robotic approach might have led some surgeons to prefer the open operation for high-risk cases. Despite the growing body of literature reporting on favourable outcomes of robot-assisted RP in high-risk PCa [18], including the evidence that the use of intra-operative visual cues can compensate for lack of tactile feedback [19], the absence of well-designed randomised or head-to-head comparative studies may have generated some uncertainties among health providers. Alternatively, the limited uptake of robot-assisted surgery in several countries in Europe, especially in CEE (where open RP has the highest preference in our survey), may be attributable to health economic reasons. These countries have a lower budget assigned for healthcare and, thus, their interest in investing in costly technology may be limited. Our survey did not evaluate whether a robot was available at the respondent's institution, and, if available, whether a robot-assisted approach would have been favoured over the open one for high-risk PCa; however, in one US study, it has been shown that hospitals with availability of robotic surgery increased the RP volume by $\sim 30$ cases/year, while hospitals without robotic facilities decreased the volume by $\sim 5$ cases/year [20]. It might thus be anticipated that with increasing dissemination of robotic systems across Europe, these figures will change in favour of the robotic approach.

Fourth, PLND at the time of RP was performed by virtually all urologists; however, despite the currently accepted recommendation to systematically perform an extended PLND in patients with high-risk PCa [4,5], the use of an extended 
Fig. 8 Preferences for administration of $\mathbf{A}$, adjuvant and $\mathbf{B}$, salvage androgen deprivation treatment after radical prostatectomy for high-risk prostate cancer. Numbers are proportions of respondents per geographical area. Total refers to the entire European sample $(N=585)$.

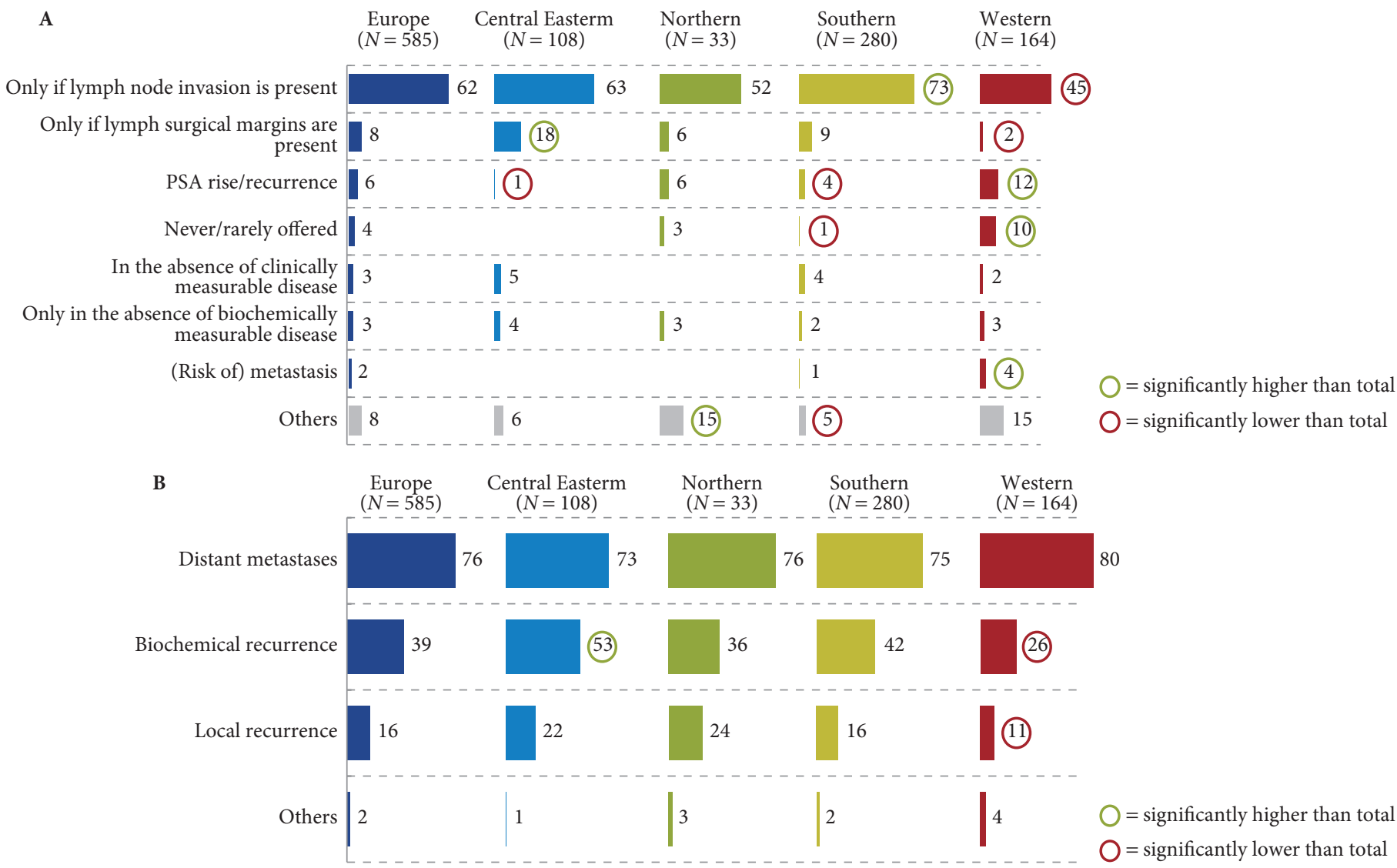

and standard template was equally split among surgeons. Possible reasons not to perform an extended PLND may be the absence of convincing high-level evidence as to its curative role [21], and the potential for greater morbidity [22]. Also, more than one third of respondents considered that the number of nodes removed was not relevant. This is consistent with the findings of recent publications $[23,24]$, rather emphasising the value of an accurate dissection of anatomically determined pelvic regions. Additionally, roughly half of respondents of the minority who routinely perform frozen sections during PLND reported they abandon surgery in the case of lymph node metastases. This practice is reminiscent of the old belief that patients with PCa with positive lymph nodes have a systemic disease with poor prognosis, but increasing contemporary evidence suggests that RP with extended PLND is an important component in multimodal therapy of locally advanced PCa with the potential to improve survival [25].

Fifth, there was much heterogeneity in the definition of disease recurrence after primary treatment and in the use of adjuvant and salvage therapies. The lack of consensus on biochemical and local recurrence has direct implications in the subsequent choice of adjuvant and salvage treatment, as disease progression rates may vary by up to $35 \%$ depending on the definition used [1]. Only 53\% of respondents would offer adjuvant EBRT in the presence of positive surgical margins, while $43 \%$ would consider salvage EBRT after any rise in PSA level $\geq 0.2 \mathrm{ng} / \mathrm{mL}$ confirmed by a second measurement. Accumulating evidence from randomised trials indicates that adjuvant EBRT to the prostate bed after RP favourably influences the course of disease compared with observation in men with adverse pathological features [26]. No similar high-level evidence for salvage EBRT is available, however, although in some observational studies a substantial proportion of patients with high-risk PCa showed a durable response to salvage EBRT, provided that it was administered at the earliest evidence of BCR [27]. Potential concerns discouraging surgeons from adopting an immediate EBRT policy are the detrimental effect on the functional status (i.e. recovery of urinary continence and potency) [26,27], and the risk of overtreatment for many patients who would have never recurred or progressed in the absence of EBRT [26].

Sixth, considerable geographical differences do exist with regard to various aspects of high-risk PCa management. A 
similar scenario has been described among urologists of North America [28], as well as for the management of other malignancies, such as breast cancer [29]. Possible reasons are disparities in national health systems, the availability of diagnostic and therapeutic technologies and cultural background.

The present study has some limitations. First, it is subject to volunteer bias, as the majority of invited participants did not respond. Also, there was no pre-determined sample size that was equally representative of each of the four geographical areas in Europe. Respondents from SE accounted for roughly half of the European sample, with the potential to unbalance the results. Furthermore, differences in management patterns within the geographical area were not assessed. It might well be that significant differences in management exist even within the same country. Second, we only focused on urologists and did not include radiation and medical oncologists, which could have led to specialty bias. In a recent national US survey on physician attitudes towards localized PCa [30], in fact, it was shown that urologists and radiation oncologists had different treatment preferences and perception of oncological and functional outcome, with implications potentially relevant to treatment recommendations and patient counselling. Third, the majority of the respondents were urologists practising in academic and tertiary referral centres, which may have different resources and technologies available compared with those practising at regional/county hospitals. Fourth, reasons for individual choice of one treatment over another (e.g. patient age, comorbidity and preferences, or surgeon/hospital experience) were not investigated for either the primary tumour or its recurrence.

In conclusion, with the limitation of a low response rate, the present study is the first international survey to evaluate patterns of management of high-risk PCa among urologists in Europe. Although definition of high-risk PCa was uniform and consistent with common guidelines, the use of RP as the initial step of a multiple-treatment approach, the adoption of a standard PLND template as frequently as an extended one, and a deferred EBRT policy for disease recurrence represent deviations in practice from the guideline recommendations, and warrant further exploration. Moreover, considerable geographical variations in patterns of staging and treatment were observed. This phenomenon may be attributable to the absence of high-level evidence, coupled with variability in local medical infrastructure availability. Whether these differences in standard of care might affect oncological outcomes requires further study.

\section{Conflict of Interest}

None declared.

\section{References}

1 Yossepowitch O, Eggener SE, Serio AM et al. Secondary therapy, metastatic progression, and cancer-specific mortality in men with clinically high-risk prostate cancer treated with radical prostatectomy. Eur Urol 2008; 53: 950-9

2 Cooperberg MR, Cowan J, Broering JM, Carroll PR. High-risk prostate cancer in the United States, 1990-2007. World J Urol 2008; 26: 211-8

3 Walz J, Joniau S, Chun FK et al. Pathological results and rates of treatment failure in high-risk prostate cancer patients after radical prostatectomy. BJU Int 2011; 107: 765-70

4 European Association of Urology Guidelines on Prostate Cancer. Latest update available at: http://www.uroweb.org. Accessed March 29, 2014

5 National Comprehensive Cancer Network Clinical Practice Guidelines in Oncology, Prostate Cancer. Version 1. 2014. Latest update available at: http://www.nccn.org. Accessed March 29, 2014

6 Alcaraz A, Burgos FJ, Cózar JM et al. Prostate cancer in Spain: from guidelines to clinical practice. BJU Int 2011; 108: 61-6

7 Stensvold A, Dahl AA, Fosså SD et al. Clinicians' use of guidelines as illustrated by curative treatment of prostate cancer at a comprehensive cancer center. Acta Oncol 2011; 50: 408-14

8 Bauvin E, Soulié M, Ménégoz F et al. Medical and non-medical determinants of prostate cancer management: a population-based study. Eur J Cancer 2003; 39: 2364-71

9 Krupski TL, Kwan L, Afifi AA, Litwin MS. Geographic and socioeconomic variation in the treatment of prostate cancer. J Clin Oncol 2005; $23: 7881-8$

10 Cooperberg MR, Broering JM, Carroll PR. Time trends and local variation in primary treatment of localized prostate cancer. J Clin Oncol 2010; $28: 1117-23$

11 ICC/ESOMAR Code on Market and Social Research. Available at: http://www.esomar.org. Accessed March 29, 2014

12 Bastian PJ, Boorjian SA, Bossi A et al. High-risk prostate cancer: from definition to contemporary management. Eur Urol 2012; 61: 1096-106

13 Abdollah F, Sun M, Thuret R et al. A competing-risks analysis of survival after alternative treatment modalities for prostate cancer patients: 1988-2006. Eur Urol 2011; 59: 88-95

14 Kibel AS, Ciezki JP, Klein EA et al. Survival among men with clinically localized prostate cancer treated with radical prostatectomy or radiation therapy in the prostate specific antigen era. J Urol 2012; 187: 1259-65

15 Nepple KG, Stephenson AJ, Kallogjeri D et al. Mortality after prostate cancer treatment with radical prostatectomy, external-beam radiation therapy, or brachytherapy in men without comorbidity. Eur Urol 2013; 64: $372-8$

16 Sun M, Sammon JD, Becker A et al. Radical prostatectomy vs radiotherapy vs observation among older patients with clinically localized prostate cancer: a comparative effectiveness evaluation. BJU Int 2014; 113: $200-8$

17 Lee EK, Baack J, Duchene DA. Survey of practicing urologists: robotic versus open radical prostatectomy. Can J Urol 2010; 17: 5094-8

18 Yuh B, Artibani W, Heidenreich A et al. The role of robot-assisted radical prostatectomy and pelvic lymph node dissection in the management of high-risk prostate cancer: a systematic review. Eur Urol 2014; 65: 918-27

19 Tewari AK, Patel ND, Leung RA et al. Visual cues as a surrogate for tactile feedback during robotic-assisted laparoscopic prostatectomy: posterolateral margin rates in 1340 consecutive patients. BJU Int 2010; 106: $528-36$

20 Makarov DV, Yu JB, Desai RA, Penson DF, Gross CP. The association between diffusion of the surgical robot and radical prostatectomy rates. Med Care 2011; 49: 333-9

21 Briganti A, Blute ML, Eastham JH et al. Pelvic lymph node dissection in prostate cancer. Eur Urol 2009; 55: 1251-65 
22 Briganti A, Chun FK, Salonia A et al. Complications and other surgical outcomes associated with extended pelvic lymphadenectomy in men with localized prostate cancer. Eur Urol 2006; 50: 1006-13

23 Dorin RP, Daneshmand S, Eisenberg MS et al. Lymph node dissection technique is more important than lymph node count in identifying nodal metastases in radical cystectomy patients: a comparative mapping study. Eur Urol 2011; 60: 946-52

24 Davies JD, Simons CM, Ruhotina N, Barocas DA, Clark PE, Morgan TM. Anatomic basis for lymph node counts as measure of lymph node dissection extent: a cadaveric study. Urology 2013; 81: 358-63

25 Gakis G, Boorjian SA, Briganti A et al. The role of radical prostatectomy and lymph node dissection in lymph node-positive prostate cancer: a systematic review of the literature. Eur Urol 2013. doi: 10.1016/j.eururo.2013.05.033 [Epub ahead of print]

26 Stephenson AJ, Bolla M, Briganti A et al. Postoperative radiation therapy for pathologically advanced prostate cancer after radical prostatectomy. Eur Urol 2012; 61: 443-51

27 Suardi N, Gallina A, Lista G et al. Impact of adjuvant radiation therapy on urinary continence recovery after radical prostatectomy. Eur Urol 2014; 65: 546-51

28 Fleshner N, Rakovitch E, Klotz L. Differences between urologists in the United States and Canada in the approach to prostate cancer. J Urol 2000; 163: 1461-6

29 Fayanju OM, Stoll CR, Fowler S, Colditz GA, Jeffe DB, Margenthaler JA. Geographic and temporal trends in the management of occult primary breast cancer: a systematic review and meta-analysis. Ann Surg Oncol 2013; 20: 3308-16

30 Kim SP, Gross CP, Nguyen PY et al. Specialty bias in treatment recommendations and quality of life among radiation oncologists and urologists for localized prostate cancer. Prostate Cancer Prostatic Dis 2014; 17: 163-9
Correspondence: Gianluca Giannarini, Urology Unit, Azienda Ospedaliero Universitaria Santa Maria della Misericordia, Piazzale Santa Maria della Misericordia 15, IT-33100 Udine, Italy.

e-mail: gianluca.giannarini@hotmail.it

Abbreviations: PCa, prostate cancer; EAU, European Association of Urology; CEE, Central-Eastern Europe; NE, Northern Europe; SE, Southern Europe; WE, Western Europe; $\mathrm{RP}$, radical prostatectomy; EBRT, external beam radiation therapy; $\mathrm{ADT}$, androgen deprivation therapy; PLND, pelvic lymph node dissection; BCR, biochemical recurrence; NCCN, National Comprehensive Cancer Network.

\section{Supporting Information}

Additional Supporting Information may be found in the online version of this article at the publisher's web-site:

Appendix S1 European survey exploring patterns of management of patients with newly diagnosed high-risk prostate cancer.

Appendix S2 Countries composing the four geographical areas in which Europe was divided for the purpose of our survey. 